\title{
THE ORBIT SPACE OF A SPHERE BY AN ACTION OF $Z_{p^{s}}$
}

\author{
STEPHEN J. WILLSON
}

\begin{abstract}
Let $X$ be a finite $\mathrm{CW}$ complex with the $Z_{p^{r}}$ homology of an $n$ sphere. Suppose $Z_{p}$, acts cellularly on $X$. The homology of the orbit space $X / Z_{p^{s}}$ with coefficients $Z_{p^{\prime}}$ is computed.
\end{abstract}

Introduction. Let $X$ be a finite $\mathrm{CW}$ complex. Denote by $Z_{m}$ the cyclic group of order $m$. If $n \mid m$, then $Z_{n}$ is naturally identified with a subgroup of $Z_{m}$. The group $Z_{1}$ is the identity group. A cellular action of $Z_{m}$ on $X$ is a cellular map $\alpha: X \rightarrow X$ such that $\alpha^{m}$ equals the identity map. If $H$ is a subset of $Z_{m}, H$ may be identified with a collection of maps $\alpha^{i}$, and the set of points in $X$ left fixed by each element of $H$ is denoted $X^{H}$. If we identify a point $x \in X$ with $\alpha(x)$, we obtain the orbit space $X / Z_{m}$. If $Z_{n} \subset Z_{m}$, then $X^{Z_{n}}$ inherits a $Z_{m / n}$ action, and $X^{Z_{n}} / Z_{m / n}$ is naturally contained in $X / Z_{m}$. The (-1)-sphere is, by definition, the empty set.

In this paper we shall assume $p$ is a prime, $X$ has the $Z_{p^{r}}$ homology of an $n$ sphere, and $Z_{p^{s}}$ acts cellularly on $X$. We shall then compute the homology of the orbit space $X / Z_{p^{s}}$. In particular, we prove the following theorem.

THEOREM A . Let $p$ be an odd prime integer, and let $r \geqslant s$. Suppose $X$ is a finite $C W$ complex with the $Z_{p^{r}}$ homology of an $n$-sphere, and $Z_{p^{s}}$ acts cellularly on $X$. Assume, for $l=0,1, \ldots, s$, that $X^{Z_{p^{(s-l)}}}$ has the $Z_{p^{r}}$ homology of a $k_{l}$-sphere (so $\left.k_{0} \leqslant k_{1} \leqslant \cdots \leqslant k_{s}=n\right)$. Then $H_{i}\left(X / Z_{p^{s}} ; Z_{p^{r}}\right)$ equals $Z_{p^{r}}$ for $i=0 ; 0$ for $1 \leqslant i<k_{0}+2 ; Z_{p}$ for $k_{0}+2 \leqslant i<k_{1}+2 ; \ldots ; Z_{p^{j}}$ for $k_{j-1}+2 \leqslant i<k_{j}$ $+2 ; \ldots ; Z_{p^{s}}$ for $k_{s-1}+2 \leqslant i<k_{s}=n ; Z_{p^{r}}$ for $i=n ; 0$ for $i>n$.

The restriction that $p$ be odd is for convenience. In fact, one needs only that for each $i$ either $k_{i}=k_{i+1}$ or $k_{i} \leqslant k_{i+1}-2$; this property is well known if $p$ is odd. If $p=2$ and for some $j, k_{j}=k_{j+1}-1$, the formulas in Theorem A need modification; in this case the change of groups is delayed by one, so that $H_{k_{j}+2}\left(X / Z_{p^{s}} ; Z_{p^{r}}\right)$ is set equal to the group (already computed) $H_{k_{j}}\left(X / Z_{p^{s}} ; Z_{p^{r}}\right)$. Thus, if $s=5, k_{0}=1, k_{1}=3, k_{2}=4, k_{3}=5, k_{4}=7, k_{5}=9$, we obtain that $H_{i}\left(X / Z_{p^{s}} ; Z_{p^{r}}\right)$ equals 0 for $1 \leqslant i \leqslant 2 ; Z_{p}$ for $3 \leqslant i \leqslant 6 ; Z_{p^{4}}$ for $7 \leqslant i$ $\leqslant 8 ; Z_{p^{r}}$ for $i=9$.

The assumption that $X^{Z_{p^{i}}}$ has the $Z_{p^{r}}$ homology of a sphere is no restriction at all; this is an easy application of the Smith theorem and the Universal Coefficient Theorem. (It is not hard to see that if $Y$ has the $Z_{p^{r}}$ homology of

Presented to the Society, August 19, 1975 under the title Applications of an equivariant universal coefficient theorem; received by the editors February 28, 1975.

AMS (MOS) subject classifications (1970). Primary 55C35; Secondary 57E25.

(c) American Mathematical Society 1976 
an $n$-sphere, then $H_{n}(Y ; Z)$ contains a free summand $Z$; and $H_{i}(Y ; Z)$ contains no $p$-torsion for any $i$.)

We note that if one desires $H_{i}\left(X / Z_{p^{s}} ; Z_{p^{j}}\right)$ where $1 \leqslant j<s$, by an easy application of the Universal Coefficient Theorem, one need only tensor the group obtained in Theorem $\mathrm{A}$ with $Z_{p^{j}}$; one uses the fact that $Z_{p^{j}} \otimes Z_{p^{k}}$ $=Z_{p^{m}}$ where $m$ is the minimum of $j$ and $k$.

Moreover, if $q$ is a prime other than $p$ and $R=Z_{q^{r}}$ for some $r$ or $R$ is the field of rational numbers, then $H_{i}\left(X / Z_{p^{s}} ; R\right)=H_{i}(X ; R)^{Z_{p^{\prime}}}$, the subgroup of $H_{i}(X ; R)$ left fixed by the homology map induced by $\alpha$. (See, for example, [1, p. 37].) Hence our result completely determines the integral homology groups of $X / Z_{p^{s}}$.

The proof of Theorem A is based on our paper [4]. We shall briefly summarize here the salient features of that paper: Suppose $R$ is a commutative ring. One may construct from $R$ and the group $Z_{p^{s}}$ a ring 9 , called the isotopy ring. Suppose $h_{i}(X, A)$ is an equivariant homology theory defined for pairs of finite CW complexes with cellular $Z_{p^{s}}$ actions; assume $h_{i}\left(Z_{p^{s}} / K\right)=0$ for all $i>0$ and all subgroups $K$ of $Z_{p^{s}}$; and that $h_{0}\left(Z_{p^{s}} / K\right)$ is an $R$ module for each $K$. Then one may construct a left 9 module $M$ with the following property: For any pair $(X, A)$ of finite $\mathrm{CW}$ complexes with cellular $Z_{p^{s}}$ action, there is a first quadrant spectral sequence with $E_{m, n}^{2}=\operatorname{Tor}_{m}^{q}\left({ }_{G} H_{n}(X, A ; \mathscr{q}), M\right)$ which converges to $h_{*}(X, A)$. Here ${ }_{G} H_{n}(X, A ; \mathscr{G})$ is a particular right 9 module with the property that, as an $R$ module, ${ }_{G} H_{n}(X, A ; 9)=\oplus H_{n}\left(X^{K}, A^{K} ; R\right)$, where the summation runs over all subgroups $K$ of $Z_{p^{s}}$.

An example. In the proof of Theorem $A$, it will be convenient to have an

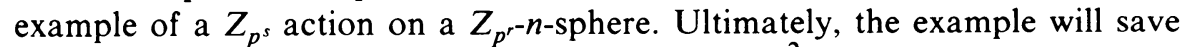
us some messy algebra in the computation of the $E^{2}$ terms of various spectral sequences.

Let $\rho_{j}$ denote the complex numbers with the (linear) action $g \cdot v$ $=\exp \left((2 \pi i) / p^{(s-j)}\right) v$, where $g$ generates $Z_{p^{s}}$. Then

$$
m_{0} \rho_{0} \oplus m_{1} \rho_{1} \oplus \cdots \oplus m_{s} \rho_{s} \quad\left(\text { for } m_{i} \geqslant 0\right)
$$

is a vector space of dimension $2\left(m_{0}+m_{1}+\cdots+m_{s}\right)$ over the reals. Let $X$ denote its unit sphere, so that $X$ is an $n=2\left(m_{0}+\cdots+m_{s}\right)-1$-sphere. Let $k_{l}=2\left(m_{s-l}+m_{s-l+1}+\cdots+m_{s}\right)-1$ for $0 \leqslant l \leqslant s$. Then $X$ has a $Z_{p^{s}}$ action, and $X^{Z_{p^{(s-l)}}}$ is a $k_{\Gamma}$ sphere.

Steenrod and Epstein [3, p. 67] show how to obtain a convenient cell decomposition of $X$ so that $g$ becomes a cellular map. If, for example, the unit sphere $S$ of $\rho_{s-2} \oplus m_{s-1} \rho_{s-1} \oplus m_{s} \rho_{s}$ has been given a cell decomposition already and $m_{s-2}>1$, then we obtain a cell decomposition of the unit sphere $T$ of $2 \rho_{s-2} \oplus m_{s-1} \rho_{s-1} \oplus m_{s} \rho_{s}$ as follows: The sphere of $\rho_{s-2}$ has a cell decomposition with $p^{2} 0$-cells $e^{0}, g e^{0}, \ldots, g^{p^{2}-1} e^{0}$ and $p^{2} 1$-cells $e^{1}, g e^{1}, \ldots$, $g^{p^{2}-1} e^{1}$. For the $i$-cells of $T, i \leqslant k=2\left(m_{s-1}+m_{s}+1\right)-1$, the dimension of $S$, we use the cells of $S . T$ has $p^{2}(k+1)$-cells, namely $S * g^{i} e^{0}$ (the join); and $p^{2}(k+2)$-cells, namely $S * g^{i} e^{1}$.

In this manner we obtain a cell decomposition for $X$ with $1 i$-cell $e^{i}$ if $0 \leqslant i \leqslant k_{0}$; with $p i$-cells $e^{i}, g e^{i}, \ldots, g^{p-1} e^{i}$ if $k_{0}+1 \leqslant i \leqslant k_{1} ; \ldots$; with $p^{m} i$-cells $e^{i}, g e^{i}, \ldots, g^{p^{m}-1} e^{i}$ if $k_{m-1}+1 \leqslant i \leqslant k_{m}$. It is easy to see that 
$g\left(g^{j} e^{i}\right)=g^{j+1} e^{i}$ where $j+1$ is reduced modulo the relevant power of $p$. Moreover if $i$ is even and $k_{m-1}+1<i \leqslant k_{m}$, then

$$
\partial\left(g^{j} e^{i}\right)=\sum_{l=0, p^{m}-1} g^{l} e^{i-1} .
$$

If $i=k_{m-1}+1$,

$$
\partial\left(g^{j} e^{i}\right)=\sum_{l=0, \ldots, p^{m-1}-1} g^{l} e^{i-1} .
$$

If $i$ is odd and $k_{m-1}+1<i \leqslant k_{m}$, then

$$
\partial\left(g^{j} e^{i}\right)=g^{j+1} e^{i-1}-g^{j} e^{i-1} .
$$

Note that by suspending the above $X$, we may ensure that $k_{0}$ be even if desired. We obtain readily the following facts about this $X$.

Lemma 1. Let $r \geqslant s$. Suppose $0 \leqslant k_{s-2}<k_{s-1}<k_{s}$. Then

$H_{i}\left(X / Z_{p^{s}}, X^{Z_{p}} / Z_{p^{s-1}} ; Z_{p^{r}}\right)$ equals 0 for $i \leqslant k_{s-1} ; Z_{p^{r}}$ for $i=k_{s-1}+1 ; Z_{p^{s}}$ for $k_{s-1}+2 \leqslant i<k_{s}$.

$H_{i}\left(X / Z_{p^{s}}, X^{Z_{p^{2}} / Z_{p^{s-2}}} ; Z_{p^{r}}\right)$ equals 0 for $i \leqslant k_{s-2} ; Z_{p^{r}}$ for $i=k_{s-2}+1 ; Z_{p^{s-1}}$ for $k_{s-2}+2 \leqslant i<k_{s-1}$.

Proof. A simple exercise. Q.E.D.

\section{Proofs.}

LEMMA 2. Let $r \geqslant s$. Suppose $X$ is a finite $C W$ complex with the $Z_{p^{r}}$ homology of an $n$-sphere. Let $Z_{p^{s}}$ act cellularly on $X$, so that $X^{Z_{p}}$ has the $Z_{p^{r}}$ homology of a $k$-sphere, $0 \leqslant k<n$. Then $H_{i}\left(X / Z_{p^{s}}, X^{Z_{p}} / Z_{p^{s-1}} ; Z_{p^{r}}\right)$ equals 0 for $0 \leqslant i \leqslant k$; $Z_{p^{r}}$ for $i=k+1 ; Z_{p^{s}}$ for $k+2 \leqslant i<n ; Z_{p^{r}}$ for $i=n ; 0$ for $i>n$.

Proof. Let $\Gamma$ be the left 9 module corresponding to the homology theory

$$
h_{i}(X, A)=H_{i}\left(X / Z_{p^{s}}, X^{Z_{p}} / Z_{p^{s-1}} \cup A / Z_{p^{s}} ; Z_{p^{r}}\right) .
$$

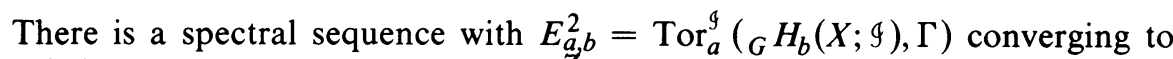

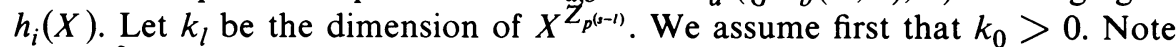
that $E_{a, b}^{2}=0$ for $0<b<k_{0}$. Hence, for $b<k_{0}, E_{b, 0}^{2}=h_{b}(X)$ for any $X$ with the assumed properties. Using Lemma 1 , and noting that $E_{b, 0}^{2}$ is independent of $k_{0}$ (as long as $0<k_{0}$ ), we see $E_{b, 0}^{2}=0$ for all $b$.

Now, since $E_{a, b}^{2}=0$ for $k_{0}<b<k_{1}$, it follows $E_{a, k_{0}}^{2}=h_{a+k_{0}}(X)$ for any such $X$. Using our example, $E_{a, k_{0}}^{2}=0$ for all $a$. Continuing in this manner, we see $E_{a, b}^{2}=0$ for $a<k_{s-1}=k$. But $E_{a, b}^{2}=0$ for $k<b<k_{s}=n$. Hence $E_{a, k}^{2}=h_{a+k}(X)$ for any such $X$. By Lemma 1, using the independence of $H_{k}(X ; 9)$ from $n$, we see $E_{0, k}^{2}=0 ; E_{1, k}^{2}=Z_{p^{r}} ; E_{i, k}^{2}=Z_{p^{s}}$ for $i \geqslant 2$. Thus we obtain the lemma for $i<n$. It is well known that $h_{i}(X)=0$ for $i>n$. (See, for example, [1, p. 43].) Finally, $E_{n, 0}^{2}={ }_{G} H_{n}(X ; 9) \otimes \Gamma=Z_{p^{r}}$, and $d$ : $E_{n-k+1, k}^{2} \rightarrow E_{n, 0}^{2}$ becomes $d: Z_{p^{s}} \rightarrow Z_{p^{r}}$. If $d$ were not one-to-one, then $h_{n+1}(X)$ would not equal zero. Hence $E_{n, 0}^{\infty}=Z_{p^{r-s}}, E_{n-k, k}^{\infty}=Z_{p^{s}}$, and the arder of $h_{n}(X)$ is $p^{r}$. The case $r=1$ would show that $h_{n}(X)=Z_{p}$. From this 
fact, a consideration of cases and the Universal Coefficient Theorem, using the fact that $h_{n+1}(X)=0$, shows $h_{n}(X)=Z_{p^{r}}$.

Minor modifications in the above argument yield the result if $k_{0}=k_{1}$ $=\cdots=k_{j}=-1$ for some $j<s-1$. Q.E.D.

Lemma 3. Let $r \geqslant s$. Let $X$ be as in the statement of Theorem A. Suppose

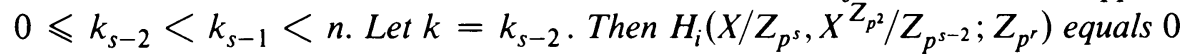
for $0 \leqslant i \leqslant k ; Z_{p^{r}}$ for $i=k+1 ; Z_{p^{s-1}}$ for $k+2 \leqslant i \leqslant k_{s-1}$.

Proof. The proof is completely analogous to that of Lemma 2, using $h_{i}(X, A)=H_{i}\left(X / Z_{p^{s}}, X^{Z^{2}} / Z_{p^{s-2}} ; Z_{p^{r}}\right)$, a corresponding left 9 module $\Gamma$, and the fact that

$$
E_{0, k_{s-1}}^{2}={ }_{G} H_{k_{s-1}}(X ; 9) \otimes_{9} \Gamma=0 .
$$

Q.E.D.

Proof of Theorem A. We prove Theorem A by induction on $s$. If $s=1$, we let $\theta$ be the left 9 module corresponding to $h_{i}(X, A)=H_{i}\left(X / Z_{p}, A / Z_{p} ; Z_{p^{r}}\right)$. The spectral sequence converging to $h_{i}(X)$ has $E_{a, b}^{2}=0$ for $b \neq 0, k_{0}, k_{1}$. Assuming $0<k_{0}<k_{1}=n$ we see $E_{a, 0}^{2}=h_{a}(X)$ for any such $X$, if $0 \leqslant a$ $<k_{0}$. Hence by use of our example, $E_{0,0}^{2}=Z_{p^{r}} ; E_{a, 0}^{2}=0$ for $a>0$. Hence $E_{a, k_{0}}^{2}=h_{a+k_{0}}(X)$ for any such $X$, if $a+k_{0}<k_{1}$. By use of our example, $E_{0, k_{0}}^{2}=E_{1, k_{0}}^{2}=0 ; E_{a, k_{0}}^{2}=Z_{p}$ for $a \geqslant 2$. Finally,

$$
E_{0, k_{1}}^{2}={ }_{G} H_{k_{1}}(X ; 9) \otimes \theta=Z_{p^{r}} .
$$

We obtain our result immediately for $0 \leqslant i<n$ and $i>n$; for the case $i=n$ we argue as at the end of Lemma 2 . The case $k_{0} \leqslant 0$ is handled similarly.

We now assume Theorem A for $s-1$ and prove it for $s$. Hence $H_{i}\left(X^{Z_{p}} / Z_{p^{s-1}} ; Z_{p^{r}}\right)$ is known by induction. In particular,

$$
H_{i}\left(X^{\left.Z_{p} / Z_{p^{s-1}} ; Z_{p^{r}}\right)=0 \text { for } i>k_{s-1}} .\right.
$$

Yet $H_{i}\left(X / Z_{p^{s}}, X^{Z_{p}} / Z_{p^{s-1}} ; Z_{p^{r}}\right)=0$ for $0 \leqslant i \leqslant k_{s-1}$ by Lemma 2 . The long exact sequence for the pair $\left(X / Z_{p^{s}}, X^{Z_{p}} / Z_{p^{s-1}}\right)$ then implies

$$
H_{i}\left(X / Z_{p^{s}} ; Z_{p^{r}}\right)=H_{i}\left(X / Z_{p^{s}}, X^{Z_{p}} / Z_{p^{s-1}} ; Z_{p^{r}}\right) \text { for } i \geqslant k_{s-1}+2
$$

and

$$
H_{i}\left(X / Z_{p^{s}} ; Z_{p^{r}}\right)=H_{i}\left(X^{Z_{p} / Z_{p^{s-1}}} ; Z_{p^{r}}\right) \text { for } 0 \leqslant i \leqslant k_{s-1}-1 .
$$

This yields our result for all $i$ except $i=k_{s-1}$ and $i=k_{s-1}+1$.

The same long exact sequence implies that

$$
\begin{aligned}
0 & =H_{k_{s-1}+1}\left(X^{Z_{p}} / Z_{p^{s-1}} ; Z_{p^{r}}\right) \rightarrow H_{k_{s-1}+1}\left(X / Z_{p^{s}} ; Z_{p^{r}}\right) \\
& \rightarrow Z_{p^{r}} \rightarrow Z_{p^{r}} \rightarrow H_{k_{s-1}}\left(X / Z_{p^{s}} ; Z_{p^{r}}\right) \rightarrow 0
\end{aligned}
$$

is exact. Hence $H_{k_{s-1}+1}\left(X / Z_{p^{s}} ; Z_{p^{r}}\right)$ and $H_{k_{s-1}}\left(X / Z_{p^{s}} ; Z_{p^{r}}\right)$ are both cyclic of the same order. 
Assume that $k_{s-2}<k_{s-1}$. Since $p$ is odd, by [2, p. 129] $k_{s-2} \leqslant k_{s-1}-2$.

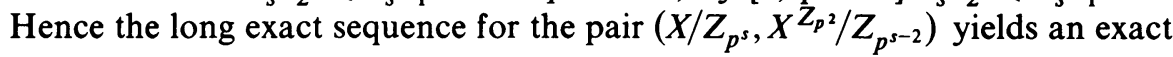
sequence

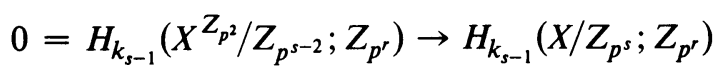

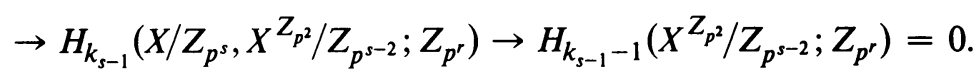

Hence, by Lemma 3,

$$
H_{k_{s-1}}\left(X / Z_{p^{s}} ; Z_{p^{r}}\right)=Z_{p^{s-1}}
$$

and our theorem follows.

If $k_{s-2}=k_{s-1}$ but $k_{s-3}<k_{s-1}$, one deals similarly with the exact sequence

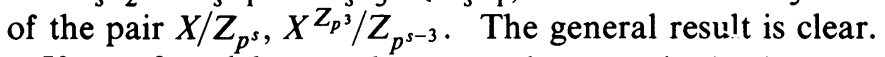

If $p=2$ and $k_{s-2}=k_{s-1}-1$, then we obtain the exact sequence

$$
\begin{aligned}
0 & \rightarrow H_{k_{s-1}}\left(X / Z_{p^{s}} ; Z_{p^{r}}\right) \rightarrow Z_{p^{r}} \rightarrow H_{k_{s-2}}\left(X^{Z_{p^{2}}} / Z_{p^{s-2}} ; Z_{p^{r}}\right) \\
& =Z_{p^{r}} \rightarrow H_{k_{s-2}}\left(X / Z_{p^{s}} ; Z_{p^{r}}\right)=Z_{p^{s-2}} \rightarrow 0
\end{aligned}
$$

Hence, in this case,

$$
H_{k_{s-1}}\left(X / Z_{p^{s}} ; Z_{p^{r}}\right)=Z_{p^{s-2}}=H_{k_{s-2}}\left(X / Z_{p^{s}} ; Z_{p^{r}}\right)
$$

Q.E.D.

\section{REFERENCES}

1. A. Borel et al., Seminar on transformation groups, Ann. of Math. Studies, no. 46, Princeton Univ. Press, Princeton, N.J., 1960. MR 22 \# 7129.

2. G. E. Bredon, Introduction to compact transformation groups, Academic Press, New York, 1972.

3. N. E. Steenrod and D. B. A. Epstein, Cohomology operations, Ann. of Math. Studies, no. 50, Princeton Univ. Press, Princeton, N.J., 1962. MR 26 \# 3056.

4. S. J. Willson, Equivariant homology theories on G-complexes, Trans. Amer. Math. Soc. 212 (1975), 155-171.

Department of Mathematics, Iowa State University, Ames, Iowa 50011 